\title{
May the force be with you
}

\author{
It may be thought that force is the province of physicists and structural engineers, but it is also \\ important in many other areas of research.
}

Force equals mass times acceleration $F=m a-$ is one of the best known equations in science. Moreover, it has the virtue of relating three things that, at first blush, seem to be fairly obvious and intuitive, although force can be quoted in a baffling array of units: newtons (the SI unit), dynes, kiloponds and poundals, not forgetting the sthène, which is equal to 1,000 newtons.

Particle physicists like to talk of the four fundamental forces - gravity, electromagnetism and the strong and weak nuclear forces - and the ongoing struggle to unify these into a single force, thus completing an exercise that started when Maxwell unified electric and magnetic phenomena. At the time of writing the electromagnetic and weak forces have been unified, and this electroweak force and the strong nuclear force are both described by quantum field theories, although they have yet to be unified. Gravity, however, remains aloof, described by the general theory of relativity, which is a classical rather than a quantum theory - not that this matters if you want to be sure that bridges and buildings do not fall down.

Gravity can be ignored at the nanoscale, where different manifestations of the electromagnetic force dominate. These forces are most notable in the plethora of atomic force microscopy techniques - chemical force microscopy, electrostatic force microscopy, magnetic force microscopy and so forth ${ }^{1,2}$ - that are available for the study and manipulation of surfaces (see also page 307). Force also has a fundamental role in biology: cellular motility, for example, is driven by molecular-scale forces ${ }^{3}$, and cells also rely on the mechanosensing of external forces to communicate with their environment ${ }^{4}$. Recent years have seen much progress in the field of single-molecule force spectroscopy, where techniques such as atomic force microscopy are used to explore the influence of force on biologica molecules and enzymatic activity ${ }^{4}$.

There is also growing interest in mechanochemistry and the deliberate use of mechanical forces to influence chemical reactions (not withstanding the discovery of fire being an early highlight in the history of mechanochemical phenomena). For a chemical reaction to occur the reactant molecules need to overcome an energy barrier, and countless chemists have spent their careers devising ways to do this with, among other things, catalysts, light, heat and electric fields.

\section{Some very deep thinkers have questioned the usefulness of the notion of force.}

In 2007, in a mechanochemical tour de force, Jeffrey Moore and coworkers at the University of Illinois at Urbana-Champaign (UIUC) showed that mechanical force could be used to initiate a reaction in benzocyclobutene molecules that normally would not happen ${ }^{5}$. In simple terms, the UIUC team attached polymer chains to the molecules, turned on an ultrasound source, and watched as mechanical stress was transmitted through the chains to the benzocyclobutene, causing a carbon-carbon bond to break. Moreover, Moore and co-workers showed that two different isomers of benzocyclobutene could be converted into the same final product - something that is not possible with non-mechanical approaches using either heat or light alone.

Another mechanochemical leap forward occurred when Rint Sijbesma and colleagues at the Eindhoven University of Technology used mechanical force to activate a catalyst for the first time ${ }^{6}$. In the past catalysts have been activated by light or by changes in $\mathrm{pH}$ or temperature: the Eindhoven team used the shear forces generated by ultrasound (and transmitted by polymer chains) to activate metal catalysts for two different organic reactions. Moreover, they could turn the catalyst off, and then back on again. It is possible that catalysts activated by mechanical force could be combined with more traditional ones that respond to either heat or light in an effort to make materials in which different chemical reactions can be triggered independently by two (or more) different stimuli. This work is likely to have applications in green chemistry and selfhealing materials.

And on page 302 of this issue, Roman Boulatov and co-workers, also at UIUC, show how force spectroscopy can be performed on molecules that are smaller than those previously studied in these types of experiments (see also page 284). Rather than polymer chains and ultrasound, Boulatov and co-workers used stiff stilbene, a U-shaped molecule that changes shape when exposed to light of certain wavelengths. The molecule of interest is attached to both ends of the ' $U$ ' and thus experiences a mechanical force when the otherwise rigid stilbene changes shape. One of the advantages of this technique is that it allows a large number of single-molecule measurements to be performed simultaneously: when atomic force microscopes or magnetic or optical tweezers are used for force spectroscopy, molecules have to be captured and stretched on a one-by-one basis, which is incredibly time consuming.

Given the ubiquity of force in the physical and natural worlds, it might surprise readers to learn that some very deep thinkers - such as the philosopher Bertrand Russell and Nobel-prizewinning physicist Frank Wilczek - have questioned the usefulness of the notion of force, with Wilczek pointing out that "force is absent from our most advanced formulations of the basic laws. It doesn't appear in Schrödinger's equation, any reasonable formulation of quantum field theory, or in the foundations of general relativity"7. Try telling that to a molecule as it is pulled apart.

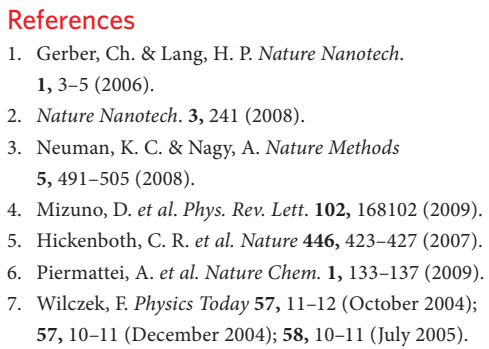

